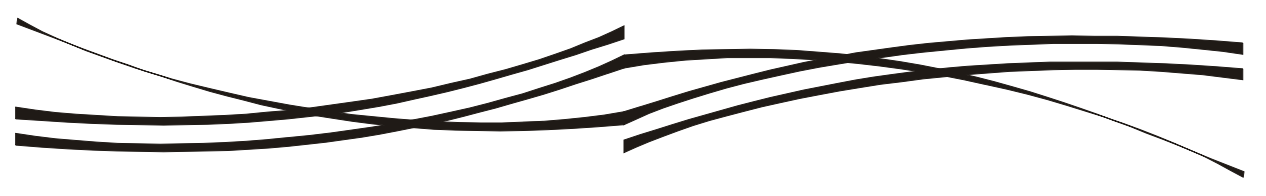

\title{
Conductividad eléctrica de carbón térmico a diferente humedad y tamaño de grano
}

\author{
Electrical conductivity of thermal coal to different \\ humidity and grain size
}

\author{
N. Y. Bautista-C. ${ }^{*}$ E. González**, \\ J. H. Bautista-Ruiz ${ }^{* * *}$, G. Peña-Rodríguez ${ }^{* * * *}$
}

\section{Resumen}

En este trabajo se reporta el desarrollo experimental para dos muestras de carbón térmico extraídas del área metropolitana de San José de Cúcuta, a las cuales se les se hizo el muestreo, cuarteo, secado y trituración con un molino mecánico. Posteriormente se determinó la composición fisicoquímica mediante el análisis próximo o inmediato, $y$, por último, se midió la conductividad eléctrica a diferentes tamaños de grano y con diferentes porcentajes de humedad, observando la relación entre el potencial (voltaje) de entrada sobre la muestra, y la respuesta o potencial de salida de la misma (método de las cuatro puntas).

\section{Palabras clave}

Carbón, análisis próximo, conductividad eléctrica.

* Escuela de Física, Grupo de Física de Materiales, Universidad Pedagógica y Tecnológica de Colombia, Tunja. E-mail: nilbaufisica@gmail.com

** Escuela de Metalurgia, Grupo Carbones y Carboquímica, Universidad Pedagógica y Tecnológica de Colombia, Tunja. E-mail: eidelmango@hotmail.com

*** Departamento de Física, Grupo de Investigación en Tecnología Cerámica, Universidad Francisco de Paula Santander, Cúcuta. E-mail: jorgebautista@ufps.edu.co

**** Departamento de Física, Grupo de Investigación en Tecnología Cerámica, Universidad Francisco de Paula Santander, Cúcuta. E-mail: ggabrielp@yahoo.com 


\begin{abstract}
This paper reports the experimental development for two thermal coal samples taken from the metropolitan area of San José de Cúcuta, to which were made sampling, quartering, drying and grinding with a mechanical grinder. Subsequently it was determined the physicochemical composition by means of near or immediate analysis, and, finally, it was measured the electrical conductivity at different grain sizes and with different percentages of humidity, observing the relationship between the potential (voltage) of input on the sample, and the response or potential output of the same(the four tips method).
\end{abstract}

\title{
Keywords
}

Coal, near analysis, electrical conductivity.

\section{Introducción}

El carbón es un combustible sólido de color negro, formado por carbono, el cual contiene componentes volátiles y no volátiles que, al quemar el carbón, quedan en forma de residuos. Este mineral se utiliza en la producción de calor dentro de hornos, en la generación de vapor, y en muchos otros sistemas térmicos como fuente principal, compitiendo con otras materias primas, tales como el petróleo, el gas natural, e incluso la madera. (Álvarez, Diez,Barriocanal\&Cimandevilla, 2005; UPTC, UIS, UFPS \& Colciencias, 2004).

El departamento de Norte de Santander está localizado en los límites con Venezuela, y hasta el año 2004 se había calculado que tiene reservas medidas de 119,69 Mt y se caracteriza por predominar la minería poco tecnificada. Este departamento se considera muy importante, debido a su ubicación geográfica donde gran parte de la producción se destina a la exportación a través del puerto de Maracaibo, en Venezuela.

Es importante caracterizar los carbones que se encuentran en esta área, con el fin de optimizarlos y sacarles el mejor provecho, ya sea para la aplicación a la industria o al desarrollo tecnológico.Con ese propósito es importante estudiar y determinar el comportamiento de la conductividad térmica, de polvos de carbón térmico, del departamento de Norte de Santander.

El propósito de este trabajo es la descripción del análisis próximo y el estudio de la ley de Ohm y conductividad eléctrica para dos minas pertenecientes a la zona metropolitana de San José de Cúcuta, a diferentes porcentajes de humedad y tres tamaños de grano. 


\section{Conductividad eléctrica de carbón térmico a diferente humedad y tamaño de grano}

La calidad de los carbones está referida a las propiedades físicas y químicas tales como humedad, cenizas, materias volátiles, carbono fijo, azufre total y poder calorífico, que son las que definirán el uso final del material (Valbuena, 2007).

Existen diferentes tipos de carbón mineral en función del grado de carbonificación que haya experimentado la materia vegetal que originó el carbón. Estos van desde la turba, que es el menos evolucionado y en el que la materia vegetal muestra poca alteración, hasta la antracita, que es el carbón mineral con mayor evolución. Esta evolución depende de la edad del carbón, así como de la profundidad y condiciones de presión, temperatura, entorno, etc., en las que la materia vegetal evolucionó hasta formar el carbón mineral.

El rango de un carbón mineral se determina en función de criterios tales como su contenido en materia volátil, contenido de carbono fijo, humedad, poder calorífico, etc. Por lo general, a mayor rango, mayor es el contenido en carbono fijo y mayor el poder calorífico, mientras que disminuyen su humedad natural y la cantidad de materia volátil. Existen varias clasificaciones de los carbones según su rango. Una de las más utilizadas divide a los carbones de mayor a menor rango en: Antracita, Bituminoso (bajo, medio o alto en volátiles), Sub-bituminoso, Lignito y Turba (Greenpeace, 2009).

\section{Conductividad eléctrica}

El voltaje medido en un conductor es proporcional a la corriente que circula por este, en un factor llamado resistencia. En la mayoría de las substancias y en un amplio intervalo de intensidades del campo eléctrico, se encuentra que la densidad de corriente es proporcional a la intensidad del campo eléctrico que la causa. A esta constante de proporcionalidad se le conoce como laconductividad del material (Purcell, 2005, p. 120 125).

Para una geometría conocida, se puede expresar la magnitud de la densidad de corriente en función de la diferencia de potencial, y por medio de esta forma se puede encontrar una expresión que relaciona explícitamente la resistencia eléctrica del material y su conductividad eléctrica (Purcell, 2005p. 120-125).

$$
\alpha=\frac{l}{R A}
$$

\section{Procedimiento experimental}

En este trabajo se utilizó carbón de las minas Las Dalias y Cerrotasajero, ubicadas en el departamento de Norte de Santander, el cual se trituró a diferentes granulometrías 
identificadas por los tamices \# 30, \# 50 , \# 60 y \# 100 (A.S.T.M E11/95, E-323, D-410). Con la malla \# 60 se determinó el análisis próximo(Tabla 1). Para los demás tamaños de grano se hicieron pruebas de ley de Ohm con porcentajes de humedad del 5,15 y 25 $\%$, respectivamente.

Los materiales e instrumentos utilizados fueron una fuente de corriente directa variable entre 0-30 v, una caja de Müller, dos multímetros, una resistencia de prueba de $1 \mathrm{M} \Omega$, una balanza, agua destilada y cables o conectores. La recolección de los datos se efectuó según lo muestra la siguiente figura:

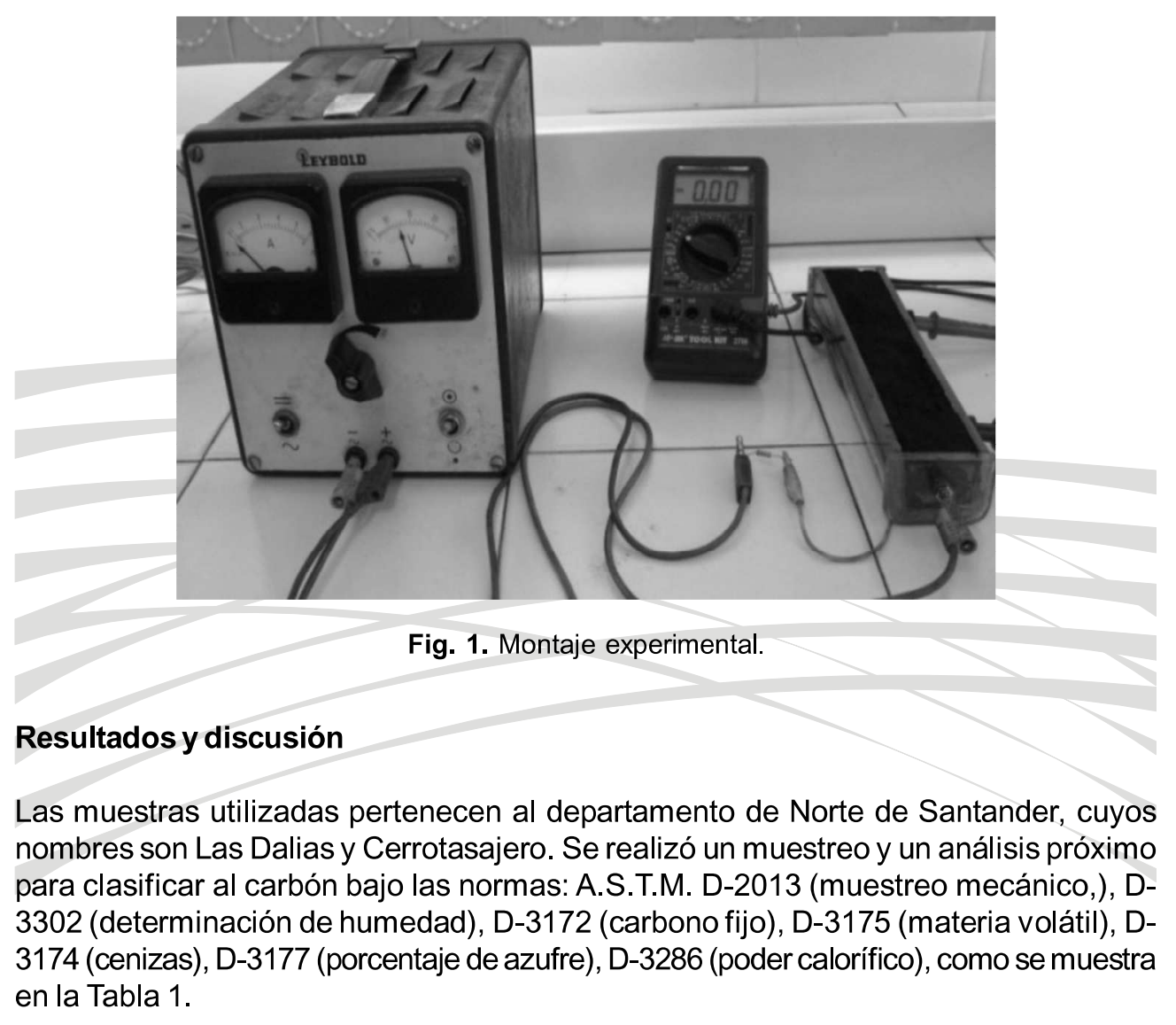




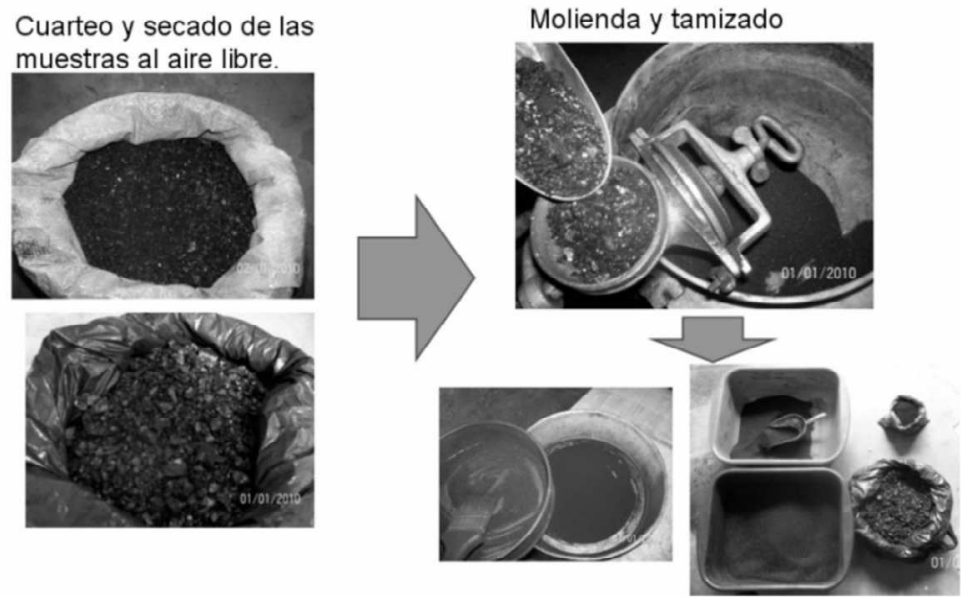

Fig. 2. Molienda y tamizado de las muestras para la determinación de la conductividad eléctrica.

Tabla 1. Determinación del análisis próximo de las muestras

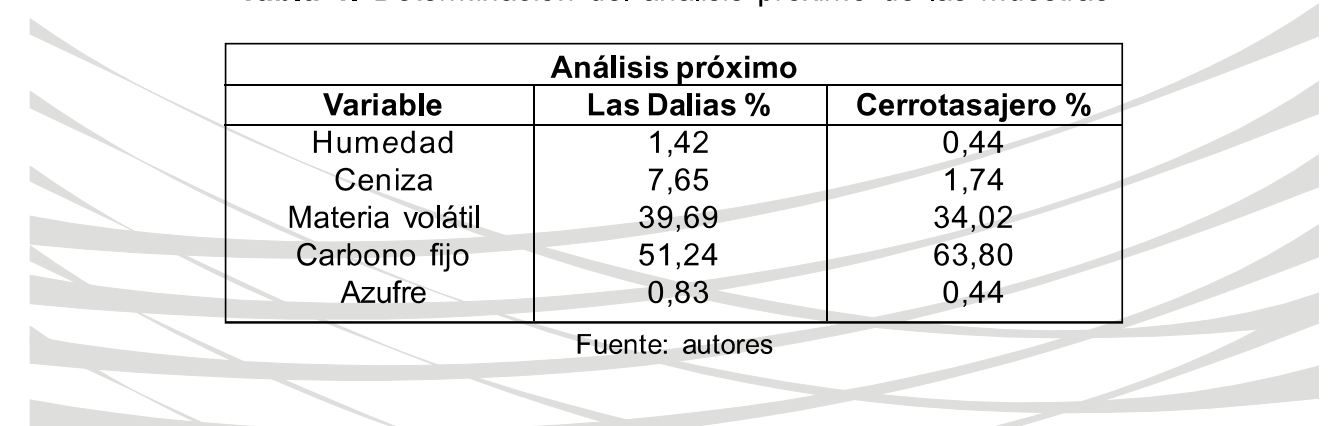

Dentro del proceso de medición de la ley de Ohm y determinación de la conductividad eléctrica, se trabajó con una caja de Müller, para identificar una geometría específica, y se utilizó una resistencia de prueba de $1 \mathrm{M} \Omega$ conectada en serie, con el fin de conocer la corriente que circula en el circuito. En las Figuras 3, 4 y 5, se presenta el comportamiento eléctrico de los carbones para las dos minas, a un tamaño de grano generado por los diferentes tamices, en donde se observa un comportamiento lineal del voltaje y la corriente, registrado en las muestras. Se anota que el valor de la pendiente es una medida de la conductividad eléctrica, la cual se ve afectada por la geometría que se utilizó. 


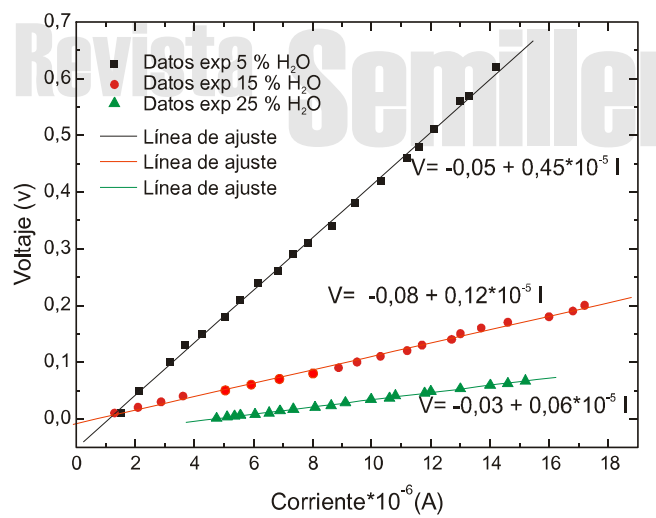

a)

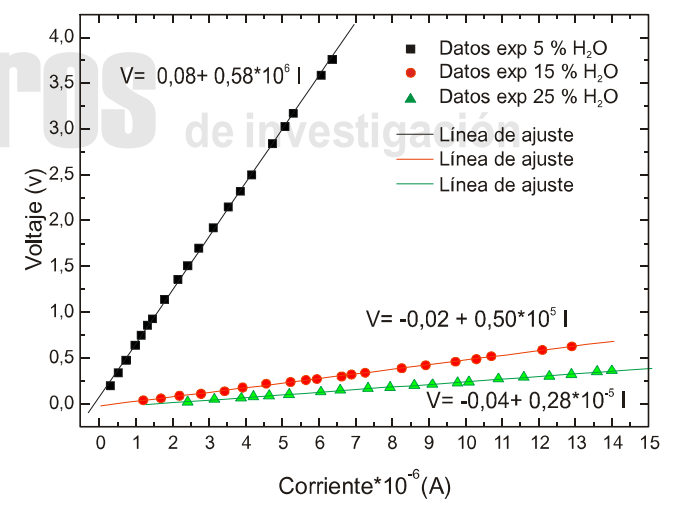

b)

Fig. 2. Conductividad eléctrica de la mina a) Las Dalias b) Cerrotasajero, para tamiz \# 30.

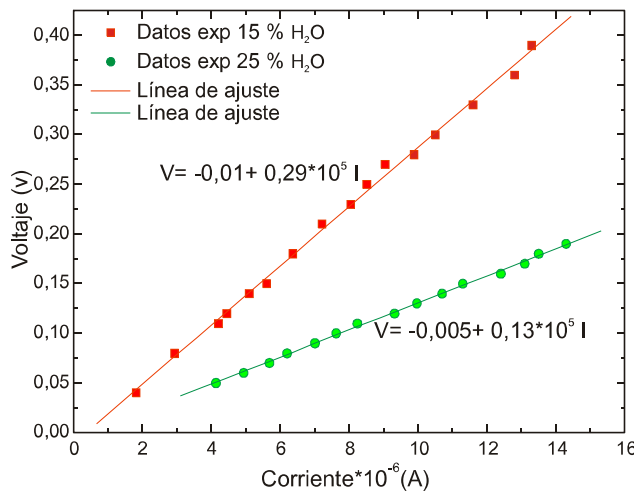

a)

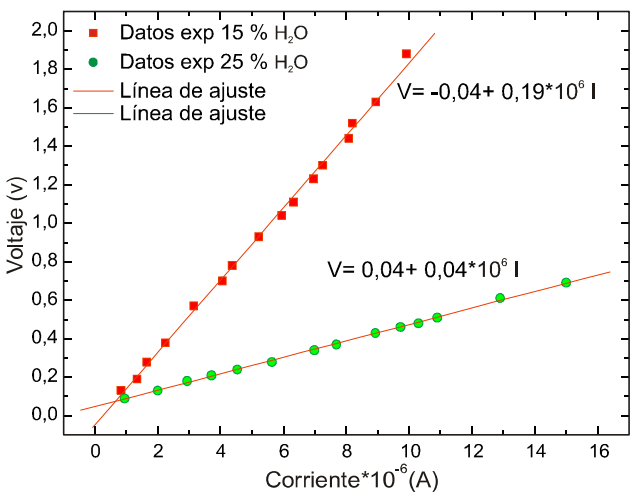

b)

Fig. 3. Conductividad eléctrica de la mina a) Las Dalias b) Cerrotasajero, para tamiz \# 50. 


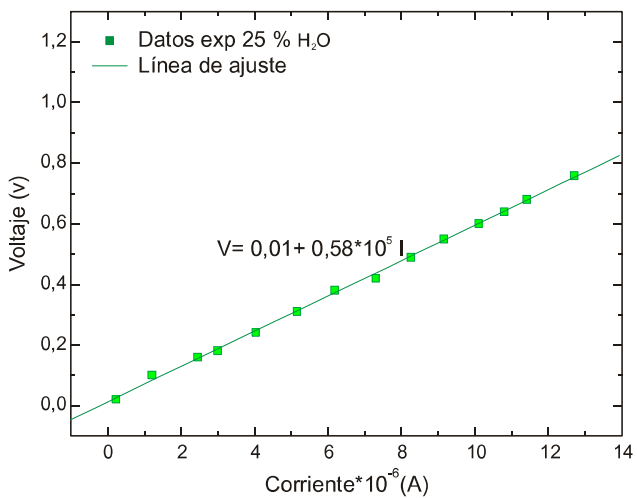

a)

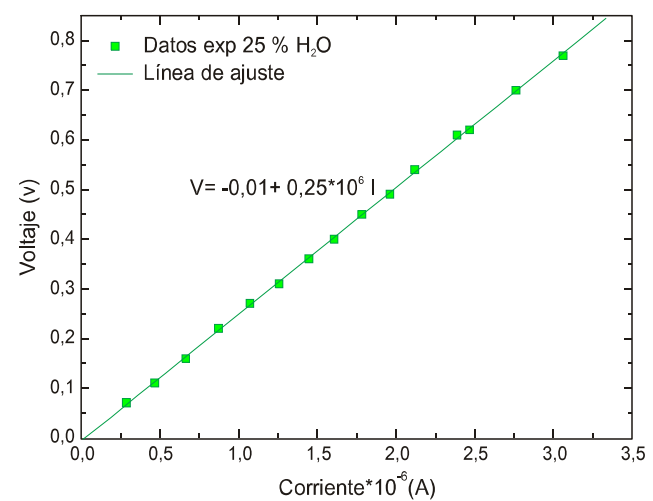

b)

Fig. 4. Conductividad eléctrica de la mina a) Las Dalias b) Cerrotasajero, para tamiz \# 100.

En las figuras se puede observar que un aumento en la humedad del polvo de carbón genera una menor pendiente en la medida, la cual representa la resistencia del material; y por medio de la ecuación (1) se puede encontrar que la conductividad eléctrica aumenta.

En las Figuras 5 y 6,se muestra una relación de la conductividad eléctrica frente al tamaño de grano para las dos minas, a una sola concentración de humedad, y se tiene mejor conductividad eléctrica para granos más gruesos, dado que la pendiente aumenta a medida que el tamaño de grano es más fino.

Esto se debe a que el agua presente en la muestra genera un mejor efecto de bordeo de los granos más grandes y no es tan absorbida por estos, generando caminos de agua por los cuales los electrones tienden a moverse más fácilmente que por los granos más finos, en donde la absorción del líquido es mayor y los caminos generados no están muy bien conectados entre sí, lo que ocasiona mayor obstáculo para que se muevan los electrones. 


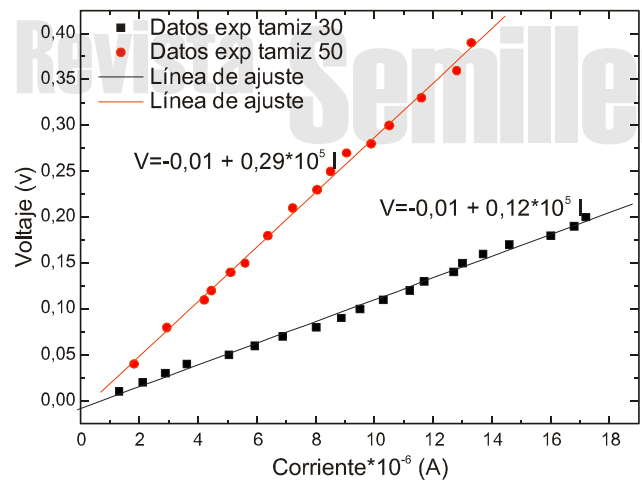

a)

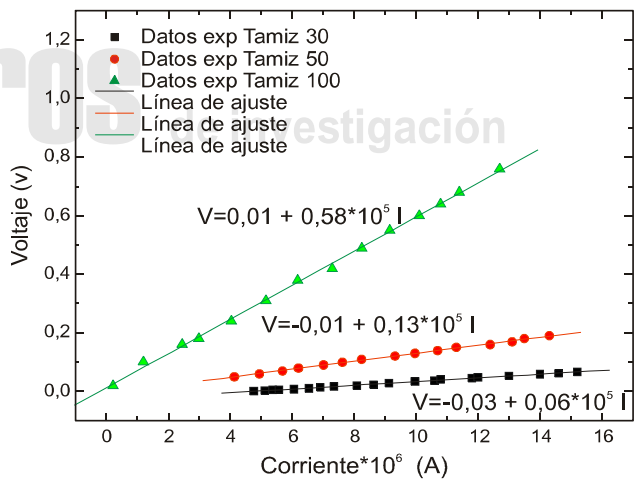

b)

Fig. 5. Comparación de la conductividad eléctrica para los diferentes tamices de la mina Las Dalias a una concentración de humedad de a) $15 \%$ y b) $25 \%$.

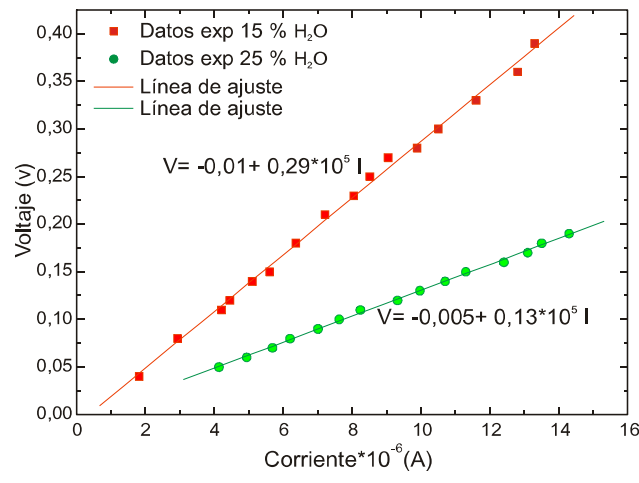

a)

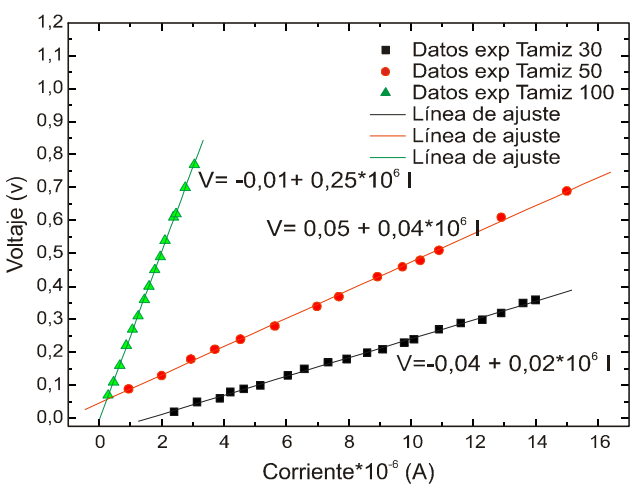

b)

Fig. 6. Comparación de la conductividad eléctrica para los diferentes tamices de la mina Cerrotasajero a una concentración de humedad de a) $15 \%$ y b) $25 \%$.

Por otro lado, al observar el valor de las pendientes, se nota que la mina con mejor conductividad eléctrica es "Las Dalias", debido a que presenta una pendiente menor a la de la otra mina. Si se tienen en cuenta los análisis próximos y se relacionan con este fenómeno, se aprecia que debido a la gran cantidad de cenizas que posee la mina se puede inferir una mejor conducción a ella, dado que estas impurezas (en su mayoría elementos) contribuyen a que los electrones tiendan a moverse con mayor facilidad, pues son más ordenados que el carbón mineral, el cual es amorfo, por lo que con estos elementos hay preferencias de recorrido que producen una mejor conductividad eléctrica. 


\section{Conclusiones}

- El fenómeno de conducción eléctrica para polvos de carbón húmedos, presenta un comportamiento de tipo lineal, en el cual se tiene un aumento en la conductividad eléctrica a medida que la muestra es más húmeda.

- Para tamaños de grano más gruesos, la conducción eléctrica es mejor que para tamaños de grano más finos, gracias a la humedad que genera caminos conductores más conectados entre sí.

- Debido a la cantidad de cenizas presentes en la mina Las Dalias, esta presenta mejor conductividad eléctrica.

\section{Lista de referencias}

Álvarez, R., Diez, M. A.,Barriocanal, C. \&Cimandevilla, J. (2005). La tecnología de producción de coque de alto horno ante el nuevo milenio. Revista de Metalurgia. Catalunya, España, V Núm. Extra, 29 - 3441 Doi: 10.3989Recuperado dehttp:// revistademetalurgia.revistas.csic.es/index.php/revistademetalurgia/article/viewArticle/994

UPTC, UIS, UFPS \& Colciencias.(2004).Selección de carbones para la aplicación del combustible CCTA en hornos de cerámica dentro de los esquemas de producción limpia y uso racional de energía. Departamentos de Boyacá, Santander y Norte de Santander.Recuperado de: http://www.umng.edu.co/www/resources/informe $\% 20$ de $\% 20$ gestion\%20colciencias\%202005.pdf

Valbuena, O. (2007). Propuesta para estudiar el efecto de la distribución de tamaño de partícula en la conductividad térmica de polvos de carbón usados en la elaboración de combustibles tipo CCTA. Tesis. Universidad Francisco de Paula Santander, Cúcuta, Colombia.

Greenpace. (2009).Carbón: combustible para el cambio climático. Proyecto central térmica Río Turbio. ( $3^{\mathrm{a}} \mathrm{Ed}$.). Buenos aires: Recuperado de: www.greenpeace.org/...I informe_base_carbon_rio_turbio.pdf

Purcell.(2005). Electricidad y magnetismo (2a Ed.). Barcelona,Editorial: Reverté, p. $120-$ 125. 